\title{
The Coupled Stratosphere-Troposphere Response to Impulsive Forcing from the Troposphere
}

\author{
THOMAS REICHLER* \\ Geophysical Fluid Dynamics Laboratory, Princeton University, Princeton, New Jersey \\ PAul J. Kushner \\ Department of Physics, University of Toronto, Toronto, Ontario, Canada \\ Lorenzo M. POLVANI \\ Department of Applied Physics and Applied Mathematics, Columbia University, New York, New York
}

(Manuscript received 31 June 2004, in final form 18 January 2005)

ABSTRACT

\begin{abstract}
A simple atmospheric general circulation model (GCM) is used to investigate the transient response of the stratosphere-troposphere system to externally imposed pulses of lower-tropospheric planetary wave activity. The atmospheric GCM is a dry, hydrostatic, global primitive-equations model, whose circulation includes an active polar vortex and a tropospheric jet maintained by baroclinic eddies. Planetary wave activity pulses are generated by a perturbation of the solid lower boundary that grow and decay over a period of 10 days. The planetary wave pulses propagate upward and break in the stratosphere. Subsequently, a zonal-mean circulation anomaly propagates downward, often into the troposphere, at lags of 30-100 days. The evolution of the response is found to be dependent on the state of the stratospheretroposphere system at the time the pulse is generated. In particular, on the basis of a large ensemble of these simulations, it is found that the length of time the signal takes to propagate downward from the stratosphere is controlled by initial anomalies in the zonal-mean circulation and in the zonal-mean wave drag. Criteria based on these anomaly patterns can be used, therefore, to predict the long-term surface response of the stratosphere-troposphere system to a planetary wave pulse up to 90 days after the pulse is generated. In an independent test, it is verified that the initial states that most strongly satisfy these criteria respond in the expected way to the lower-tropospheric wave activity pulse.
\end{abstract}

\section{Introduction}

In this study, we explore, with an idealized model, the dynamics of extratropical zonal-mean flow anomalies that are observed to propagate downward from the stratosphere into the troposphere (Baldwin and Dunkerton 1999, 2001). These signals, which we will hereafter call troposphere-stratosphere-troposphere (TST) events, have attracted attention because of their con-

* Current affiliation: Department of Meteorology, University of Utah, Salt Lake City, Utah.

Corresponding author address: Dr. Thomas Reichler, Department of Meteorology, University of Utah, 135 S 1460 E, Rm. 819 (WBB), Salt Lake City, UT 84112-0110.

E-mail: reichler@met.utah.edu nections to multiple-week tropospheric circulation forecasts (Baldwin and Dunkerton 2001; Baldwin et al. 2003b; Charlton et al. 2004) and to the long-term mean circulation response to climate change (Thompson and Solomon 2002; Gillett and Thompson 2003). The cause of TST events are planetary waves that propagate up from the troposphere, dissipate, and mix potential vorticity (PV) in the stratosphere, and bring about a mean flow and residual circulation response there (e.g., Charney and Drazin 1961; Polvani and Waugh 2004). But the dynamics of the subsequent evolution of the TST events is still unclear and raises the question of just how the stratosphere might influence the tropospheric circulation (Baldwin et al. 2003b).

Various ideas have been put forward to explain the dynamics of TST signals, including PV inversion (Black 2002; Ambaum and Hoskins 2002), eddy mean-flow interaction and downward control (Dickinson 1968; 
Haynes et al. 1991; Holton and Mass 1976; Plumb and Semeniuk 2003), and planetary wave reflection in the stratosphere (Perlwitz and Harnik 2003). Each of these ideas leads to different conclusions about the nature of the stratospheric influence. One step toward resolving these differences is to construct a relatively simple modeling framework that cleanly separates stratospheric from tropospheric influences. Without such a framework, the way the stratosphere might control, for example, the upward flux of wave activity from the troposphere that initiates the TST events remains ambiguous.

In this study, we investigate the life cycle of TST events by explicitly generating them in the lower troposphere of a relatively simple atmospheric general circulation model (GCM) of the stratosphere-troposphere system. This is in contrast to approaches in which the stratosphere is perturbed directly (e.g., Christiansen 2003; Charlton et al. 2004; Song and Robinson 2004) or in which the tropopause is perturbed and the troposphere is not modeled (e.g., Holton and Mass 1976; Plumb and Semeniuk 2003; Polvani and Saravanan 2000). Our approach can be regarded as a simple representation of observed TST events that originate in the lower troposphere (Cohen et al. 2002, their Fig. 2). The atmospheric GCM is the same dry, hydrostatic general circulation model that two of us have used in previous studies of stratosphere-troposphere dynamics (Polvani and Kushner 2002, hereafter PK; Kushner and Polvani 2004, hereafter KP). Our approach is illustrated schematically in Fig. 1: starting from a spunup atmospheric initial condition, a pulse of lower-tropospheric wave activity is generated over a period of 10 days by perturbing the solid lower boundary; the pulse propagates into the stratosphere and breaks, and the subsequent evolution of zonal flow anomalies is examined. This subsequent evolution often, but not always, yields downward propagation of the zonal flow anomalies into the troposphere, that is, complete TST events. The atmospheric response, especially long after the initial pulse is generated, is highly variable and depends strongly on the initial state of the atmosphere. We find that, although the initial driver of the perturbation is unambiguously tropospheric, the subsequent evolution of the perturbation can be influenced by stratospheric conditions. The objective of this study is to identify predictors of the long-term response. Ultimately, we wish to understand the response in light of the dynamical literature cited above.

In what follows, we first describe the model and the design of the numerical integrations (section 2) and then the results of a large ensemble of these pulse cal-

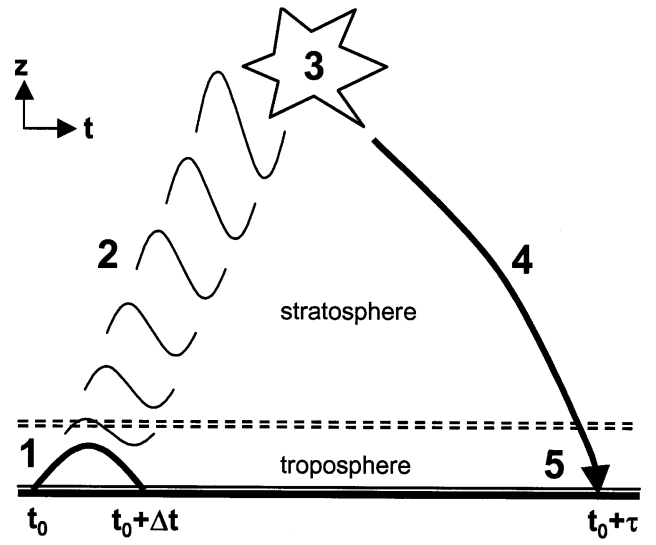

FIG. 1. Schematic illustration of the TST events simulated in this study. (1) Forced pulse of planetary waves occurring over time $\Delta t$; (2) upward-propagating waves; (3) dissipation and breaking of waves; (4) induced downward-propagating anomalies; and (5) tropospheric response at time lag $\tau>\Delta t$.

culations (section 3a). We next develop a classification technique to categorize the range of possible responses (section $3 b$ ), discuss predictors of the response (section $3 c$ ), and present a dynamical interpretation of those predictors (section 3d). We conclude with a discussion of these results and another sensitivity study (section 4). In the appendix, we provide additional technical details related to the forcing of the model.

\section{Model and design of the integrations}

\section{a. Model description}

Our model is a slightly modified version of the one used by PK and KP; the reader is referred to these papers for further details. It solves the dry, hydrostatic, primitive equations on the sphere and uses the Held and Suarez (1994, hereafter HS) prescription for Newtonian cooling in the troposphere, the PK prescription for Newtonian cooling in the stratosphere, and Rayleigh damping of the zonal and meridional winds in the planetary boundary layer and in a sponge above $0.5 \mathrm{hPa}$ (explicit formulas of the forcing functions are provided in the appendix). We use T42 spectral resolution in the horizontal and $40 \sigma$-level resolution in the vertical; the vertical levels extend from the ground to the mesosphere. The simulation represents perpetual solstitial conditions.

We note the following differences between our model and PK/KP's model. First, in our model, the tropospheric Newtonian-cooling profile matches that of HS. This is achieved by setting the parameter $\varepsilon$ in Eq. (A9), which controls the asymmetry of the tropospheric 

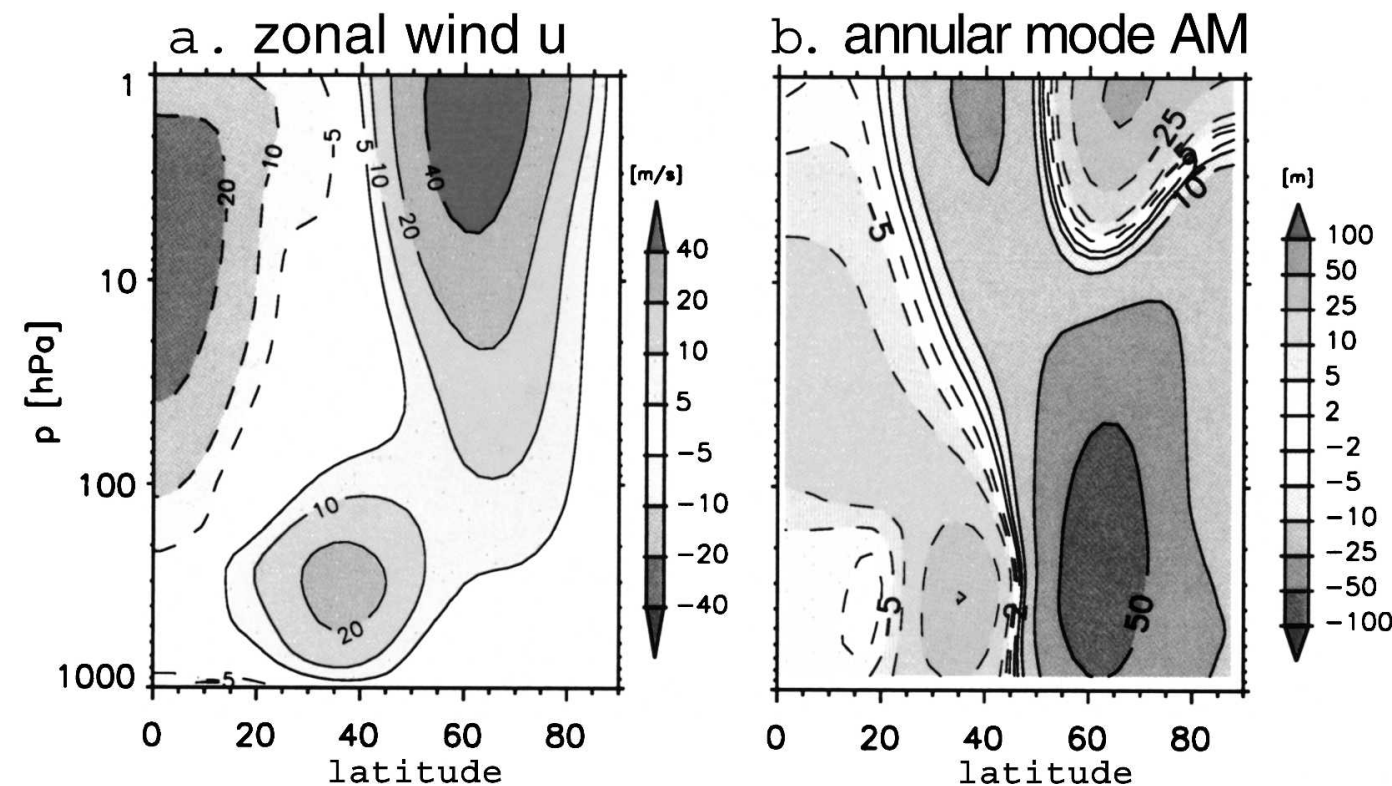

FIG. 2. Time- and zonal-mean fields as a function of latitude and pressure derived from the control experiment. (a) Zonal wind in $\mathrm{m} \mathrm{s}^{-1}$. (b) Pattern of the annular mode in $\mathrm{m}$. Here and elsewhere, negative contours are dashed.

temperature gradients between the hemispheres, to zero. Second, in the standard runs of our model, we use a stratospheric Newtonian damping time scale of 20 days, which is half the value of that used in PK, KP, and HS. This shorter damping time scale effectively strengthens the stratosphere-troposphere coupling in the model. See section 4 for further discussion of this. Finally, in our standard runs, the parameter $\gamma$, which controls the strength of the stratospheric winter hemisphere temperature gradients (PK), is set to 1 . This makes the polar vortex relatively weak compared to the $\gamma=2$ and $\gamma=4$ cases studied in PK, and thus increases the amount of wave activity absorption and stratosphere-troposphere coupling.

The zonal- and time-mean zonal winds for our control run, which is integrated for approximately 40000 days after a 1000-day spinup period, is plotted in Fig. $2 \mathrm{a}$. The model produces a fairly realistic zonal wind profile, with an active baroclinic-eddy-driven jet in the troposphere, a stratospheric polar vortex, and tropical easterlies. The maximum zonal velocities in the polar vortex reach $57 \mathrm{~m} \mathrm{~s}^{-1}$, which is close to the observed solstitial winds as shown, for example, in Fig. 1.4 of Andrews et al. (1987). As in PK and KP, the model has a flat lower boundary and forcing and dissipation terms that are zonally homogenous. Therefore, the model has no stationary eddies: the extratropical circulation is maintained by transient baroclinic eddies in the troposphere and by transient planetary eddies in the stratosphere (Scinocca and Haynes 1998).
Below, we refer to the model's annular mode (AM), whose spatial structure is shown in Fig. 2b. To obtain this figure, we first find the leading principal component time series of the daily $853-\mathrm{hPa}$ zonal-mean geopotential height poleward of $20^{\circ} \mathrm{N}$, after it has been multiplied by the square root of the cosine of latitude. The resulting first principal component time series explains $79 \%$ of the total variance. The time series is then divided by its standard deviation to yield a quantity with unit standard deviation. Figure $2 b$ shows the covariance or regression of the zonal-mean geopotential with the resulting quantity. In the troposphere and lower stratosphere, the mode consists of a meridional dipole that is similar to observations (Baldwin and Dunkerton 1999). The upper-stratospheric structure of the AM is not robust; in particular it is more sensitive to which analysis level is chosen for the EOF than the tropospheric and lower-stratospheric structure.

\section{b. Perturbation integrations}

To generate TST events, we next perform an ensemble of perturbation integrations that branch from the control run at 100-day intervals. Using the controlrun initial condition as a starting point, a pulse of upward propagating planetary wave activity is generated by specifying that the surface geopotential $\Phi_{S}$ vary as a function of space and time according to the expression

$$
\Phi_{S}(\lambda, \phi, t)=\eta(\lambda, \phi) T(t)
$$


where $\lambda$ is longitude, $\varphi$ is latitude, and $t$ is time; the shape of the perturbation is given by

$$
\eta(\lambda, \phi)= \begin{cases}A_{0} \sin ^{2}\left(\frac{\phi-\phi_{0}}{\phi_{1}-\phi_{0}} \pi\right) \cos (k \lambda) & \phi_{0} \leq \phi \leq \phi_{1} \\ 0 & \text { otherwise }\end{cases}
$$

and the temporal evolution of the surface geopotential perturbation is given by

$$
T(t)= \begin{cases}\sin ^{2} \frac{t \pi}{\Delta t} & t \leq \Delta t \\ 0 & t>\Delta t .\end{cases}
$$

The parameters we use for our standard runs are $k=1$, $A_{0}=5000 \mathrm{~m}, \varphi_{0}=40^{\circ} \mathrm{N}, \varphi_{1}=80^{\circ} \mathrm{N}$, which represents a wavenumber-1 sinusoidal perturbation centered at $60^{\circ} \mathrm{N}$, a zero mean, and a peak-to-trough displacement of $10000 \mathrm{~m}$. The time envelope parameter $\Delta t=10$ days. This parameter setting as well as the parameters that control the mean state of the polar vortex (i.e., $\gamma$ and $k_{\mathrm{st}}$, see appendix) were chosen heuristically; based on an initial sensitivity study, in which these parameter were varied, we found that this particular combination led to a reasonably strong upward propagating signal, a physically plausible stratospheric response, and a relatively large fraction of cases with well-defined TST events.

After the initial 10-day forcing period, the model is integrated with $\Phi_{\mathrm{s}}=0$ for 90 more days to investigate the response of the system to the imposed forcing. Apart from the perturbation of the lower surface for the first 10 days, the perturbation runs are identical to the control run. We conduct a total of 403 perturbation integrations; this defines our ensemble.

\section{Results}

\section{a. Ensemble-mean response}

Figure 3 shows the ensemble-mean departure of the 116-hPa geopotential height from the control-run mean at day 8 , which represents the time of the peak response at this level to the wave activity pulse from the lower troposphere. Since the surface forcing reaches its peak at day 5 , we see that it takes roughly 3 days for the waves to travel to this level. As one would expect from the structure of the forcing function, the main component of the lower-stratospheric response is a zonal wavenumber-1 perturbation that is confined roughly to latitudes north of $30^{\circ} \mathrm{N}$. The anomalies are between

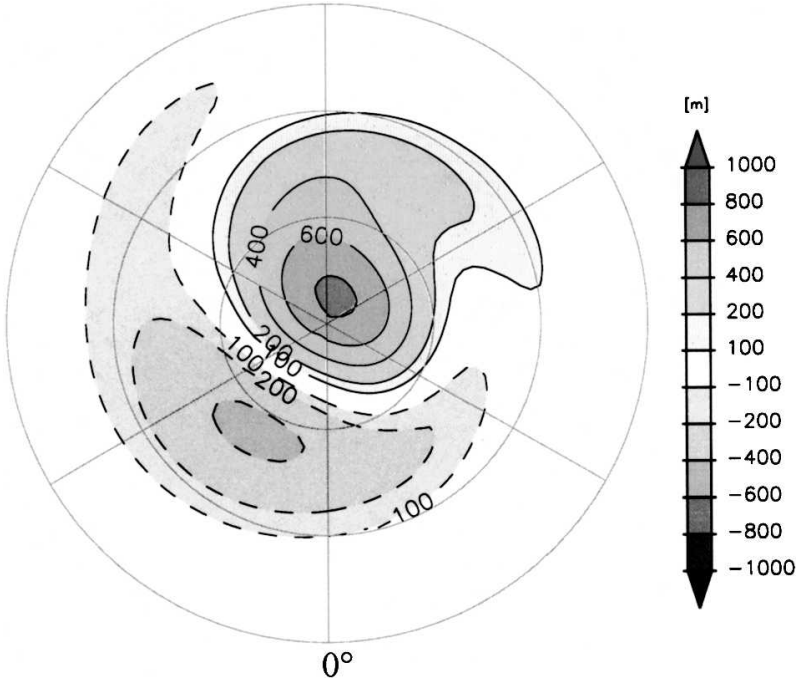

FIG. 3. The ensemble-mean departure of the 116-hPa geopotential height field from the control run during the peak response at this level (day 8).

-400 and $+600 \mathrm{~m}$, which is similar to the observed lower-stratospheric geopotential height anomalies during strong sudden warming events (e.g., Matsuno 1971; McIntyre and Palmer 1984; Andrews et al. 1987; Baldwin and Dunkerton 1989). This confirms that the externally imposed pulse of tropospheric wave activity leads to a plausible atmospheric response.

Our primary interest is in the subsequent evolution of the stratosphere-troposphere system. To show the time evolution of the response to the tropospheric wave activity pulse, we plot in Fig. 4 a measure of the geopotential response over the polar cap as a function of time and pressure. The quantity plotted is

$$
R=\frac{\langle\Phi\rangle^{\prime}(p, t)}{S_{\mathrm{ctl}}\langle(\Phi\rangle)(p)},
$$

where $\langle\Phi\rangle$ represents the zonal and $60^{\circ}-90^{\circ} \mathrm{N}$ meridional mean of the geopotential, $\langle\Phi\rangle^{\prime}$ represents the difference between the instantaneous value of $\langle\Phi\rangle$ in an individual realization and the control-run time-mean value of $\langle\Phi\rangle$, and $s_{\mathrm{ctl}}(\langle\Phi\rangle)$ represents the control-run daily standard deviation of $\langle\Phi\rangle$. We scale by units of standard deviations of the control run to indicate how large the response is compared to the day-to-day variability at each level.

Figure 4a shows the ensemble mean of $R$, and Figs. $4 \mathrm{~b}-\mathrm{d}$ show the evolution of $R$ for selected individual realizations. In the ensemble and time mean (Fig. 4a), the heights increase at almost all levels and times owing to the warming from the dissipation of the planetary wave activity. The perturbation peaks in the tropo- 

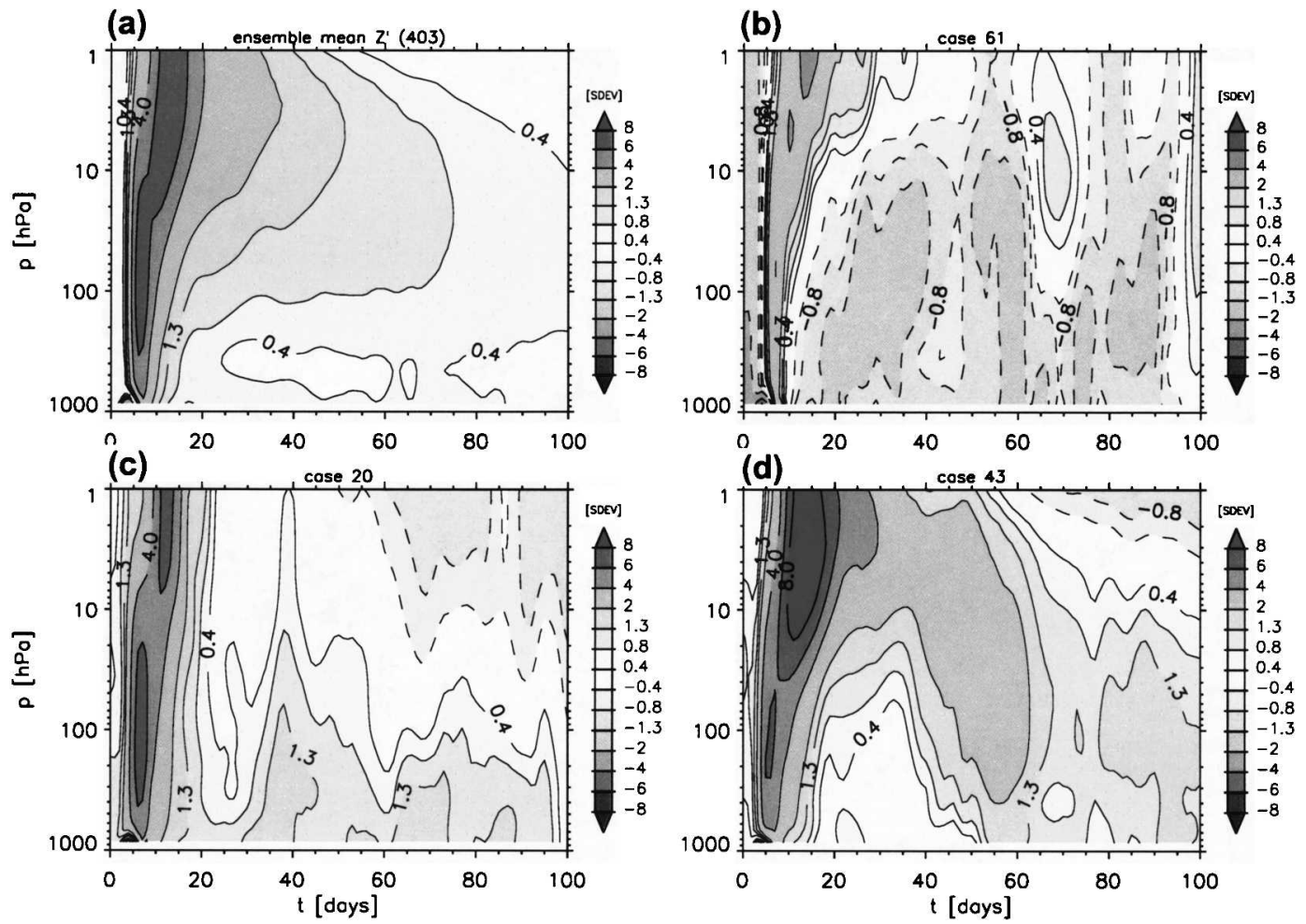

FIG. 4. The quantity $R$ in Eq. (4) as a function of time and pressure; $R$ represents normalized geopotential height anomalies averaged over the polar cap. Shown are (a) the ensemble mean over all 403 realizations, and (b)-(d) individual selected cases.

sphere and rapidly propagates into the stratosphere. After the initial forcing period (days 1-20 in the stratosphere), the stratospheric response decays to zero with a time scale consistent with the 20-day thermal damping time scale. Figure 4a gives also a subtle indication of downward propagation from the upper to the lower stratosphere, which is supported by the existence of small positive tropospheric height anomalies during days 60-80. However, a distinct stratosphere-to-troposphere downward propagating TST signal is missing.

\section{b. Classification of the realizations}

The lack of a signal that propagates back into the troposphere in the ensemble mean reflects the fact that the tropospheric evolution of the perturbation integrations is highly variable from one realization to the next. The plots of $R$ for the three individual realizations in Figs. 4b-d illustrate this spread. The timing, spatial pattern, and strength of those cases are very different, and bear little resemblance to the ensemble mean (Fig. 4a). This high variability of individual outcomes is a signature of the strong nonlinearity of the extratropical circulation and its response to external perturbations, and is one of the main reasons why we have performed an ensemble calculation with so many realizations. Only by averaging over many members does the signal to noise ratio become sufficiently large. Looking over all the ensemble members, we find that a clear downward-propagating signal, as for example exhibited by case 43 (Fig. 4d), occurs in only about one-third of all cases. Even for those cases, the timing of the tropospheric return signal is again quite variable.

Despite the large spread, we find that we are able to classify the many different outcomes of our experiments into a few basic categories. Our classification scheme is based on the time period on which the largest positive value of $R$ occurs in the troposphere, for each realization. Excluding the time period of the initial tropospheric wave pulse, we divide the time interval of each realization into early (days $25-50$ ), intermediate (days 51-75), and late (days 76-100) periods. We then calculate, for each realization, the value of the time mean and tropospheric vertical mean (specifically, the 191-853-hPa mean) $R$ in each of these three periods. If the largest positive of these values occurs in the early, intermediate, or late period, we classify the realization 

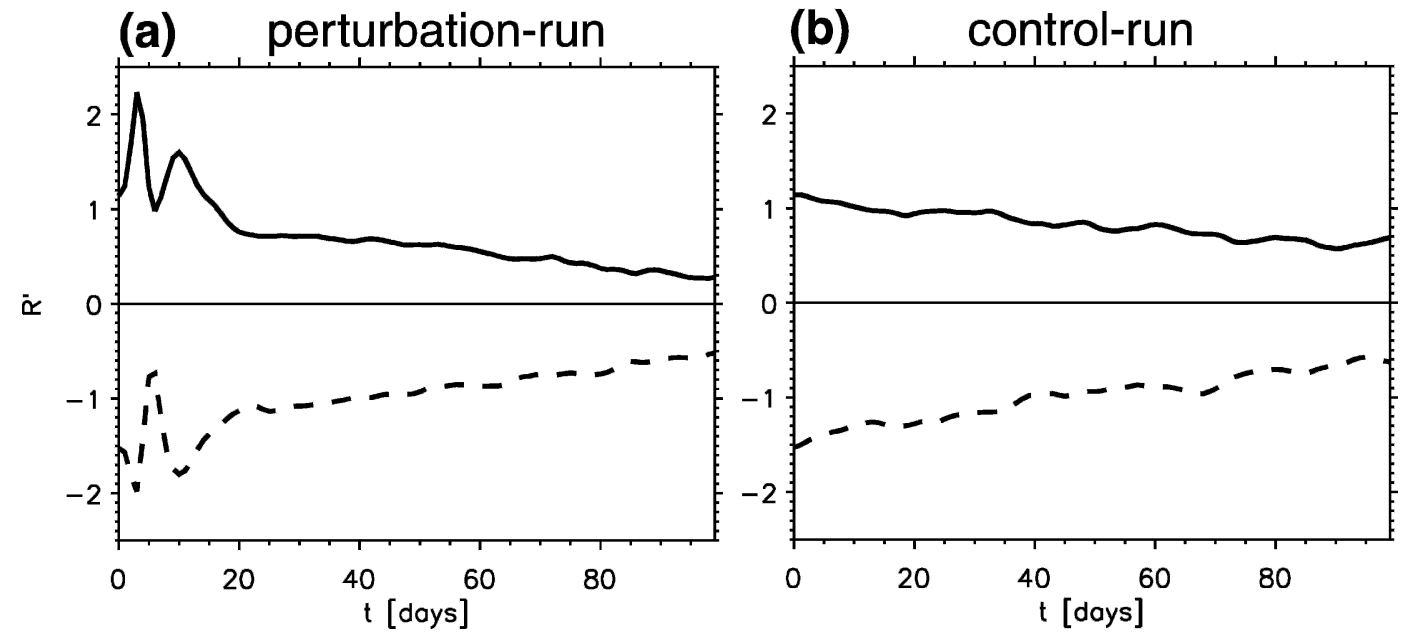

FIG. 5. The composite temporal evolution of $R$ (vertically averaged from 191 to $853 \mathrm{hPa}$ ) for realizations with a strongly negative (continuous line) or strongly positive (dashed line) annular mode index (vertically averaged from 97 to $853 \mathrm{hPa}$ ) at $t=0$ (a) for members of the perturbation ensemble, and (b) for the corresponding members of the unperturbed control run.

as "E," "I," or "L," respectively. Finally, we create composite averages over the $\mathrm{E}$, I, and L groups of realizations.

We next exclude from our classification those members of the ensemble with a large initial AM value. We do this to bypass problems with the persistent behavior of the AM in this model. This behavior is demonstrated in Fig. 5a, which shows the evolution of tropospheric vertical mean (97-853 $\mathrm{hPa}) R$ composites of those experiments that have initially a large negative $(\mathrm{AM}<$ $-1)$ or positive $(A M>1) A M$ index. One can see that the tropospheric AM that is present in the initial conditions decays very slowly over time. This and other analyses we have pursued show that the AM and other aspects of the tropospheric high-latitude variability are unrealistically persistent in this model, with a decorrelation time scale of about 100 days. This behavior is largely independent of the tropospheric wave activity pulse, as can be seen by comparing with Fig. $5 b$, which shows the tropospheric $R$ taken from the corresponding cases in the control run that are not forced by the tropospheric wave activity pulse. We have seen this behavior before in this class of simple dry atmospheric GCMs (AGCMs; KP; Garric et al. 2003; James and James 1989). We regard the simulation's AM persistence time scale of months as a model artifact because the time scale is much longer than observed AM persistence time scales of about two weeks (Baldwin et al. 2003a). This persistence interferes with our classification because it implies that a realization that includes an initially strongly positive tropospheric AM state is picked up as an L event in our classification, and an initially strongly negative AM state as an E event, independent of the perturbation or of the stratospheric response. Neither case reflects stratosphere-totroposphere propagation, and so the persistent AM signals overwhelm the more subtle downward propagating signals. We choose a threshold initial AM index magnitude of $2 / 3$ and exclude cases with initial AM magnitudes that exceed this value. This removes about half of the available realizations and leaves 201 out of the original 403 cases.

The results of the classification are shown in Fig. 6 . Figure 6a show the quantity $R$ for each of the groups (with the averaging regions in time-pressure coordinates shown for reference), and Fig. 6b shows the difference between $R$ for each of the group composites and the ensemble-mean value of $R$. The number above each panel indicates how many realizations fall in each group; the distribution of 79:63:59 is approximately equal among each of the E, I, and L categories. This classification scheme brings out better-defined TST events, particularly for the I and L cases (Figs. 6d,g); these panels shows that the tropospheric anomalies in days 80-100 are part of a large coherent pattern that starts in the upper stratosphere near the time of the initial pulse. The signal strength in the troposphere for all the composites is about 0.8 standard deviations. This translates into a geopotential height anomaly of about $40 \mathrm{~m}$, and into a change in surface pressure of about 4 $\mathrm{hPa}$.

Given the large spread in the outcome of individual experiments (Figs. 4c,d), one may ask how statistically significant the composite mean patterns of the indi- 



[SDEV]


FIG. 6. The classification of the perturbation experiments into (top) E, (middle) I, and (bottom) L cases. The classification is based on the strongest $R$ response averaged over the indicated boxes. Shown are (left) composites of $R$ as a function of time and pressure, (middle) $R$ minus $R_{\mathrm{EM}}$, the mean over the members of all three cases, and (right) $R$ divided by the standard deviation of $R$ within each group. The numbers above each panel indicate how many realizations fall into each case.

vidual groups are. To answer this question, the panels on the right-hand side of Fig. 6 show a measure of the signal to noise ratio of the quantity $R$ as a function of time and pressure. The quantity plotted is

$$
S 2 N=\frac{\langle\Phi\rangle^{\prime}(p, t)}{S_{\exp }(\langle\Phi\rangle)(p, t)},
$$

where $s_{\exp }(\langle\Phi\rangle)$ represents the member-to-member standard deviation of the perturbation experiments. The signal-to-noise ratios are approximately on the order of 1 , indicating that the mean responses are statistically relatively robust features.

The composite meridional structure of these events is shown in Fig. 7 as a sequence of 25-day time averages with the ensemble mean removed. The $\mathrm{E}$ and $\mathrm{L}$ pat- terns are structurally similar but of opposite sign, as expected from Figs. 6b,h. Anomalies whose sign match the long-term tropospheric response first appear in the upper stratosphere (days 0-24), then spread slowly into the lower stratosphere (days 25-49 and 50-74), and finally fill almost the entire atmospheric column over the polar cap (days 75-99). In the final stage, the anomalies project strongly on the AM of the model, as a comparison with Fig. $2 b$ shows.

\section{c. Predictors of the tropospheric response}

A key issue is whether predictors of the different tropospheric responses can be found in the atmospheric state at time zero, that is, immediately prior to the time of the externally imposed perturbation. The thin contouring in the top two panels of Fig. 8 shows the E 



FIG. 7. The composite meridional structure of geopotential for the (top) E and (bottom) L cases in sequences of 25-day time averages. Shown are composite geopotential heights minus the mean over the members of all three cases as a function of latitude and pressure for days $0-24,25-49,50-74$, and 75-99.

and $\mathrm{L}$ composites of the (unscaled) geopotential height differences from the ensemble mean at the initial time. For the L composite, there is a negative center in the lower stratosphere/upper troposphere that is centered around $65^{\circ} \mathrm{N}$, and a dipole structure in the upper stratosphere with a positive center around $80^{\circ} \mathrm{N}$ and a negative center around $40^{\circ} \mathrm{N}$. For the E composite, the centers are of opposite sign and at similar locations, but the upper-stratospheric centers are weaker. The zonal-mean zonal wind composites (middle panels) show that the L composite has a relatively weak and poleward shifted stratospheric polar vortex (see also Fig. 2) and a complex tropospheric structure.

Not all the features in Fig. 8 are statistically significant. The thick black contours indicate regions where the magnitudes of the anomalies exceed $10 \%$ of the climatological standard deviation of the control-run. This is a rough measure of statistical significance of the pattern features in the figure, if one assumes that the anomaly of one individual experiment is significant if it exceeds one climatological standard deviation $(\sigma)$, and that the critical standard deviation of the ensemblemean scales like $\sigma_{\mathrm{EM}} \sim \sigma /(n)^{1 / 2}$, where $n$ is the ensemble size. With roughly $n=70$ realizations in each composite, this results in a critical value of about $10 \%$. In the upper stratosphere, the relatively stronger initial anomalies in the $\mathrm{L}$ cases are more significant, by this measure, than in the $\mathrm{E}$ cases. This can also be seen in Figs. $6 \mathrm{~b}, \mathrm{~h}$ at day 0 between 1 and $10 \mathrm{hPa}$.

Since the zonal-mean eddy driving represents the tendency of the zonal-mean winds, its initial distribution might provide a predictor of the tropospheric response that is independent of the initial winds. The bottom panels of Fig. 8 show the composite EliassenPalm (EP)-flux cross sections for the E and L cases, minus the ensemble mean. The cross sections are generally noisy, especially in the troposphere, but show a 
(a)

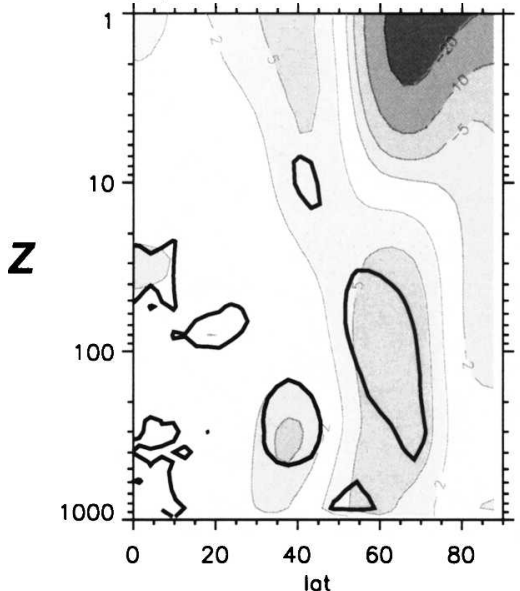

(c)

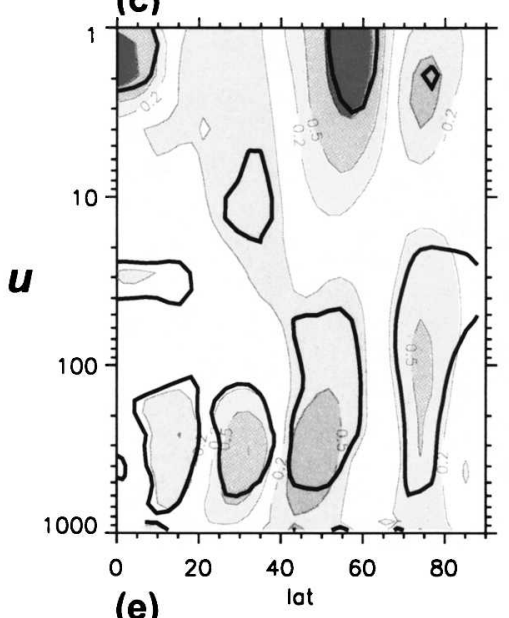

(e)

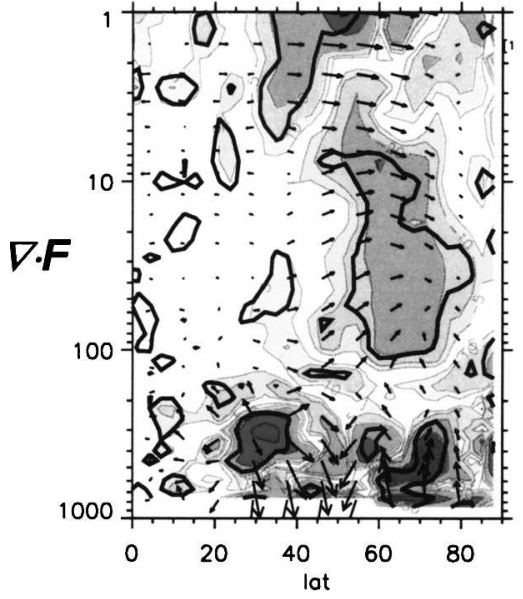

(b)

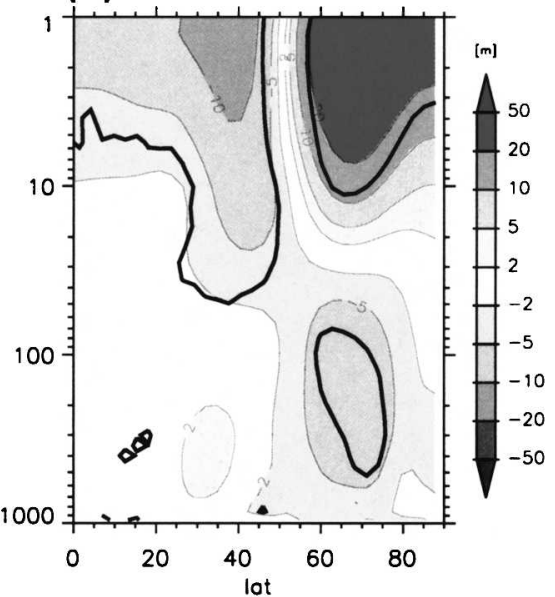

(d)

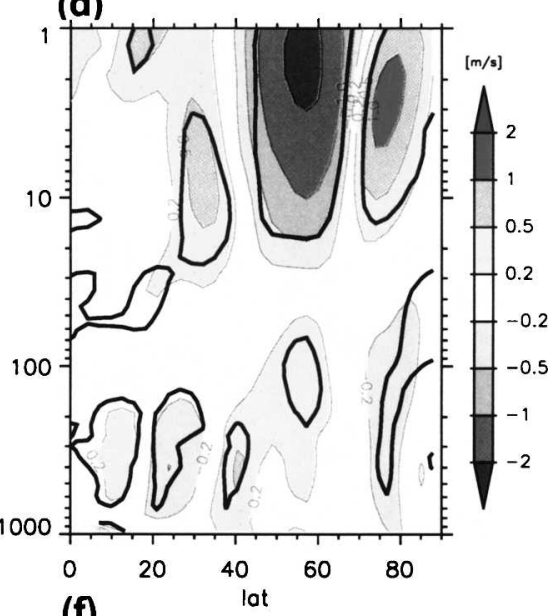

(f)

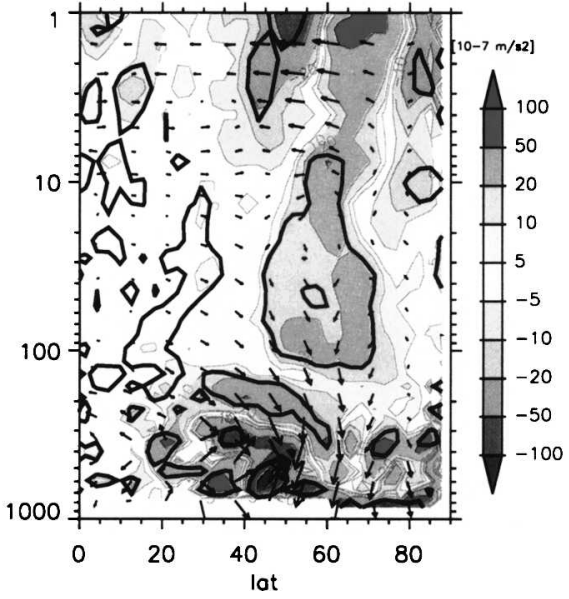

FIG. 8. The composite meridional structure of anomalies at the initial time for members of the (left) E and (right) L group. The anomalies are taken with respect to the mean over the realization of all three cases at the initial time. The thick black contours represent regions where the magnitude of the anomalies exceeds $10 \%$ of the climatological standard deviation of the control run, indicating that the anomalies are statistically significant. Shown are (top) geopotential heights in $\mathrm{m}$, (middle) zonal winds in $\mathrm{m} \mathrm{s}^{-1}$, and (bottom) EP-flux divergence (contours) and EP-flux vectors (arrows). The divergence is divided by $[a \cos (\phi)]$ to show the acceleration of the zonal-mean flow (in $10^{-7} \mathrm{~m} \mathrm{~s}^{-2}$ ). The vectors are appropriately scaled to provide an idea of the relative size and sign of the vector components. 
large and coherent positive EP-flux divergence anomaly in the extratropical lower stratosphere for the L composite and a roughly equal and opposite anomaly for the E composite.

We now test to see whether the initial anomalies in Fig. 8 can be used to predict the tropospheric outcomes in Fig. 6. We focus on the L case because the L-composite anomalies in Fig. 8 are more statistically significant. Starting from the full 403-member ensemble, we first exclude the 202 realizations that project strongly onto the AM initially (for these realizations, the best tropospheric forecast is persistence of the initial AM anomalies). We plot the composite-mean $R$, with the ensemble-mean removed, for realizations whose initial conditions show anomalously positive high-latitude EPflux divergence in the lower stratosphere (Fig. 9a), anomalously positive high-latitude geopotential in the upper stratosphere (Fig. 9b), or both (Fig. 9c). As for Fig. 8, anomalies are taken with respect to the ensemble-mean over all three groups at the initial time. The panels in Fig. 9, which are based on a priori information (i.e., the initial conditions prior to the perturbation), can be compared to Fig. 6h, which is based on a posteriori information (i.e., the known outcomes). Although one criterion alone is unable to reproduce the characteristic downward propagating patterns, both criteria together are relatively successful in predicting the L-type tropospheric response.

\section{d. An independent test of the predictors}

The favorable comparison between Figs. $6 \mathrm{~h}$ and 9c suggests that randomly chosen initial conditions that satisfy our two criteria on EP-flux divergence and upper-stratospheric wind lead fairly reliably to an $\mathrm{L}$ response. We now perform an additional test that better highlights the role of these criteria when they are very strongly satisfied. In this test, we perform a small number of additional perturbation experiments for the deliberately chosen initial states that most strongly satisfy the two criteria. We proceed as follows.

1) First, we find periods in which the 10-day running average, $60^{\circ}-90^{\circ} \mathrm{N}$ mean EP-flux divergence at 80 $\mathrm{hPa}$ takes on a particularly large value of greater than $10^{-5} \mathrm{~m} \mathrm{~s}^{-2}$. There are 29 such days in the control run. For this independent test, no preselection on AM strength is performed.

2) Of these 29 days, we select six in which the geopotential at high latitudes in the middle and upper troposphere exceeds $+200 \mathrm{~m}$. This choice is somewhat subjective, since the requirement was fulfilled by more than six initial conditions. Figure 10 presents
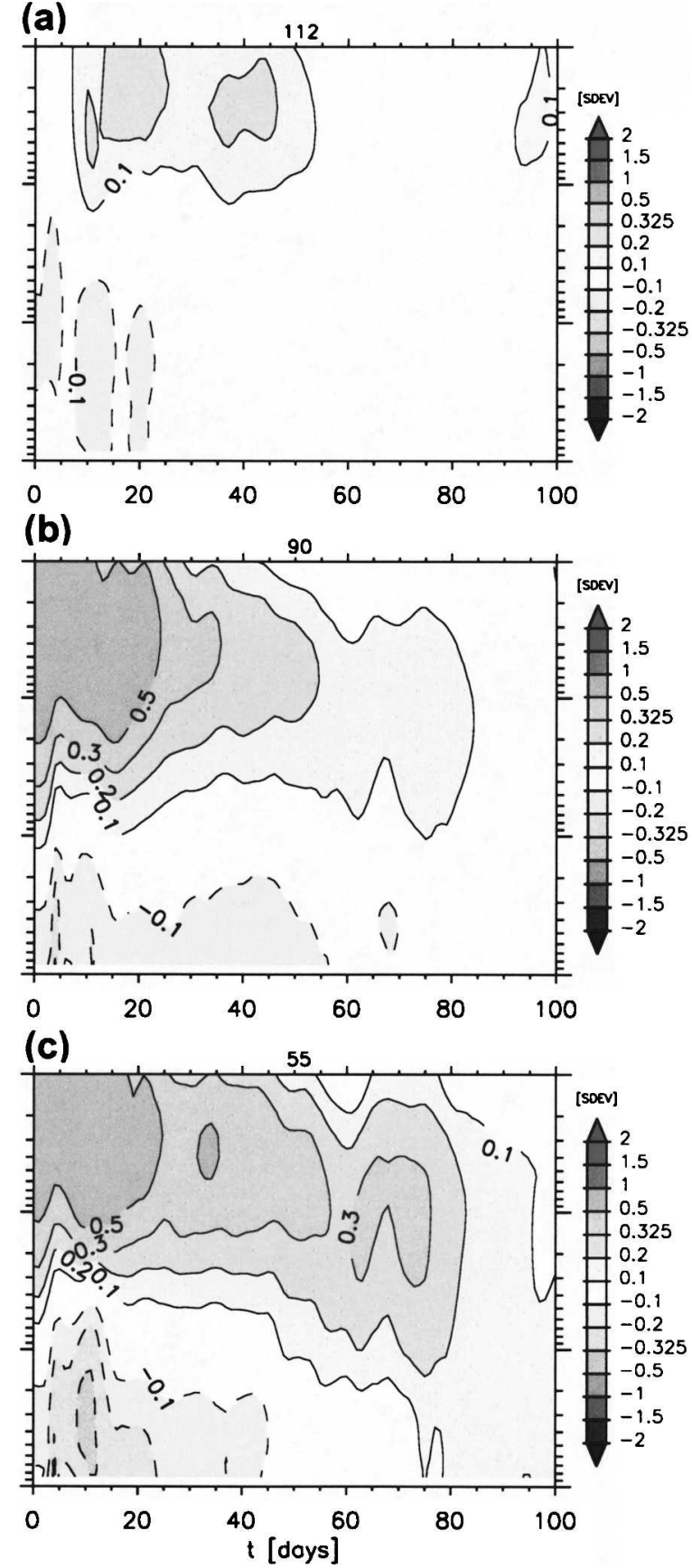

FIG. 9. Composites of $R$ as a function of time and pressure minus the ensemble-mean response for realizations with the following high-latitude $\left(60^{\circ}-90^{\circ} \mathrm{N}\right.$ mean $)$ initial conditions: (a) anomalously positive EP-flux divergence at $80 \mathrm{hPa}$, (b) anomalously positive zonal-mean geopotential at $3 \mathrm{hPa}$, and (c) both conditions.

the EP-flux, geopotential, and zonal-wind structure of these six states. Notice that the patterns are consistent with the L-composite mean of Figs. $8 \mathrm{~b}$,d,f, but that the anomaly amplitudes are approxi- 

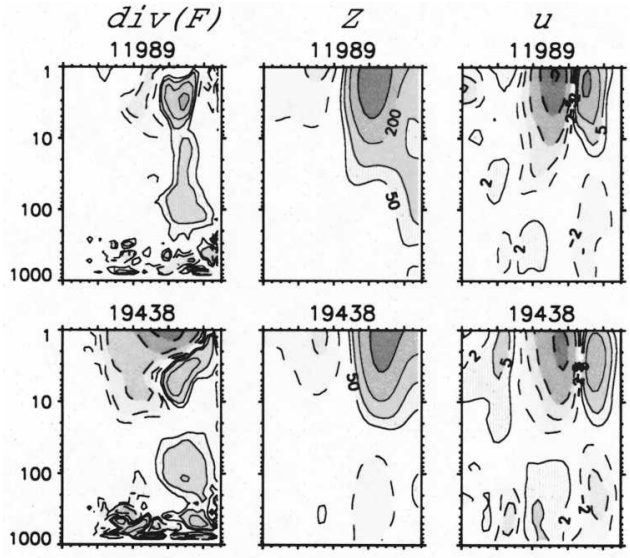

19438
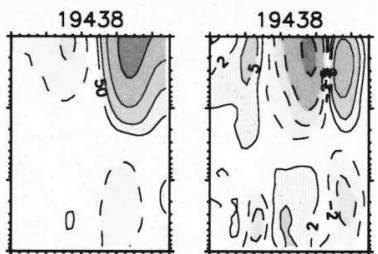

33361

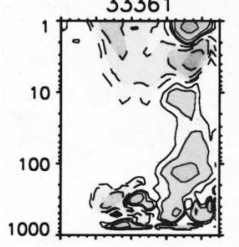

33361
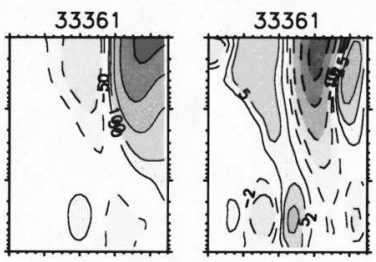

38474

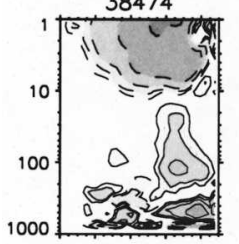

38474
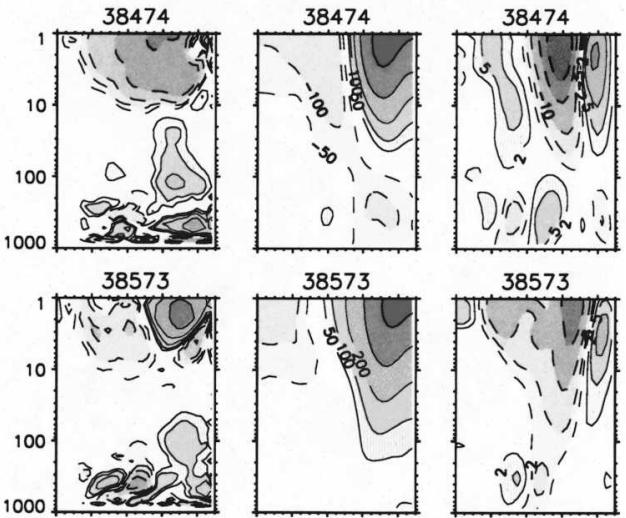

38573

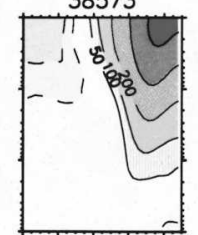

38573



39646



39646
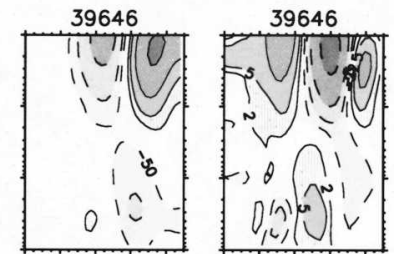

mean

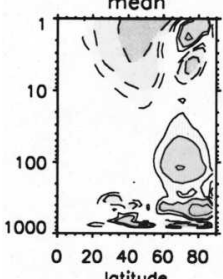

meon
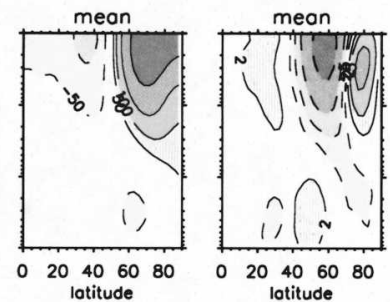

FIG. 10. The meridional structure of six initial conditions from the control run that fulfill the criteria of an L-type response. Shown are the anomalies of (left) anomalous EP-flux divergence, (middle) geopotential height, and (right) zonal wind. The anomalies are taken with respect to the climatological mean of the control run. The bottom row shows the ensemble mean of all six initial conditions. The contour levels are (left) $\pm 5, \pm 10, \pm 20, \pm 40$, $\pm 80, \pm 160 \times 10^{-6} \mathrm{~m} \mathrm{~s}^{-2},($ middle $) \pm 50, \pm 100, \pm 200, \pm 400, \pm 800$ $\mathrm{m}$, and (right) $\pm 2, \pm 5, \pm 10, \pm 20, \pm 30 \mathrm{~m} \mathrm{~s}^{-1}$. mately an order of magnitude larger for these six cases.

3) Finally, we perform our standard perturbation experiments on these six initial states, but extend the integration time from 100 to 140 days, so that more details about the response at later times are captured. The panels on the left-hand side of Fig. 11 show the outcome of the perturbation experiments in terms of the quantity $R$, and the bottom panel shows the ensemble mean of $R$ for the six runs. Each panel shows also a 20-day history of $R$ prior to the corresponding initial condition at day 0 .

The selected initial conditions indeed tend to result in an L-type response, since five out of the six experiments show well-defined stratosphere-to-troposphere signals that peak in the troposphere at around day 100. The outcomes of the individual experiments bear also a striking resemblance to downward propagating events in observations (e.g., Baldwin and Dunkerton 2001), in particular because of the familiar pattern that resembles dripping paint. The results can be compared with the panels on the right-hand side of Fig. 11, which shows the evolution of $R$ for the corresponding cases of the unperturbed control run. Interestingly, the controlrun cases show also some tendency of weak downward propagation. This is probably related to the relatively warm upper-stratospheric conditions prior to day 0 , and weak pulses of upward propagating waves shortly before day 0 . Such additional wave activity can be found in the $R$ signatures of all six cases, and leads to an upper-stratospheric warming that maximizes at day 0 . The tendency for downward propagation seems to be amplified in the perturbation experiments by imposing additional wave forcing after day 0 .

\section{e. Dynamical interpretation}

We may ask why the criteria we used in Fig. 9 would lead to a delayed tropospheric (L) response relative to the ensemble mean.

1) The L cases are favored by a weaker and poleward shifted upper-stratospheric vortex (Fig. 8d). This appears to cause more wave activity to be initially absorbed at higher levels in the stratosphere than in the ensemble mean (see Fig. 6h). This, in turn, would delay the tropospheric response if the rate of downward descent of the zonal-mean flow anomaly is roughly constant among the realizations; since the anomaly is starting at a higher level, it will take longer to descend to the surface.

2) The L cases are also favored by anomalously posi- 
Perturbation experiments

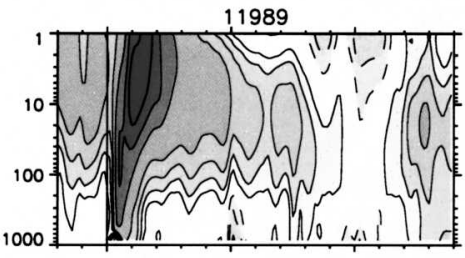

19438

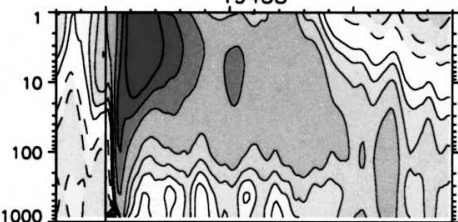

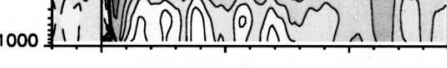



38474



38573
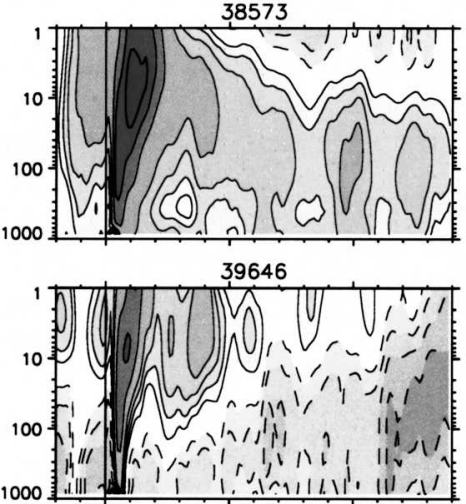

meon

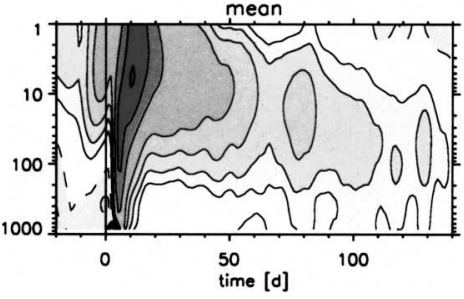

Control run

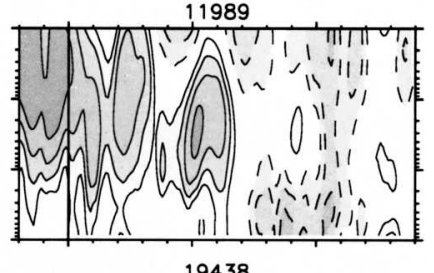

19438

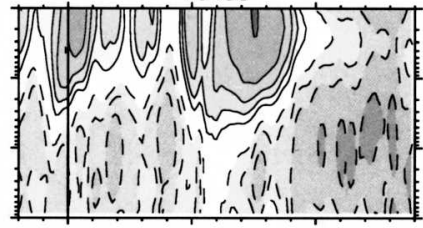

33361

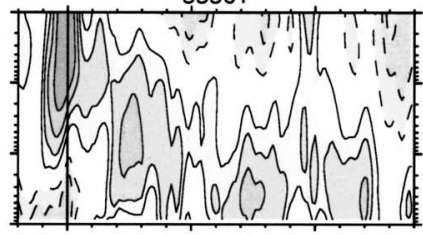

38474

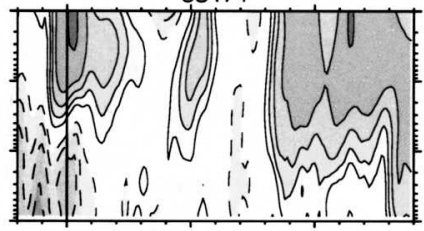

38573



39646



mean

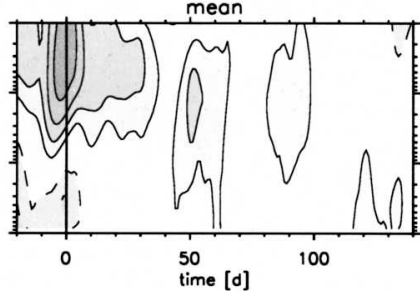

FIG. 11. The quantity $R$ as a function of time (from day -20 to +140 ) and pressure for simulations using the six selected initial conditions $(t=0)$ shown in Fig. 10. (left) Perturbation experiments starting from those initial conditions (forced from day 0 to 10), and (right) the unperturbed control run in the absence of the wave activity pulse. (bottom) The mean response of all six outcomes. Contour intervals and shading are the same as in Fig. 4. 
tive EP-flux divergence in the lower stratosphere (Fig. 8f). The EP-flux divergence signature seems to indicate that a wave activity anomaly has just propagated out of the lower stratosphere into the upper stratosphere, where it is causing eddy-induced warming. This is evident in Fig. 11, in which all the states have been preconditioned warm in the twenty days prior to time 0 . The EP-flux divergence also implies that the lower-stratospheric westerlies are set to strengthen after the main pulse is initiated. Linear theory suggests that less wave activity will be absorbed in the lower stratosphere than in the ensemble mean as a result. Thus, more wave activity will be absorbed at higher levels, which is again consistent with the impact of the weaker and polewardshifted vortex.

It is difficult to determine conclusively what the importance and dynamical interpretation of the initial tropospheric anomalies is in Figs. 8 and 10. Thus, we cannot rule out the possibility that tropospheric initial conditions exert a significant, or even a major, influence on the upward propagating pulse, beyond the AM persistence.

\section{Conclusions}

We have used an externally imposed lower-tropospheric planetary wave pulse in a simple GCM to generate troposphere-stratosphere-troposphere (TST) events in a controlled way. The ensemble-mean response, after the stratospheric warming, does not exhibit a distinct stratosphere-to-troposphere downward propagating signal. This is because the ensemble-mean averages over a broad range of responses whose characteristics emerge when we separate cases with early (E) and late (L) times of a tropospheric return signal. We use the E/L classification to illustrate the typical life cycle of the stimulated TST events (Figs. 6 and 7). This framework allows us to determine which prior atmospheric conditions predict the tropospheric response (section 3c).

We have found that realizations in which the tropospheric return signal is late (L) are favored by stratospheric conditions in which the wave activity pulse is absorbed at relatively high levels. Both the initial zonalmean winds and the eddy forcing (related to the wind tendency) provide independent information about the likelihood of a late response. Our simple explanation for the timing of the tropospheric response, illustrated schematically in Fig. 12, is that the rate of downward descent does not vary systematically among the realiza-

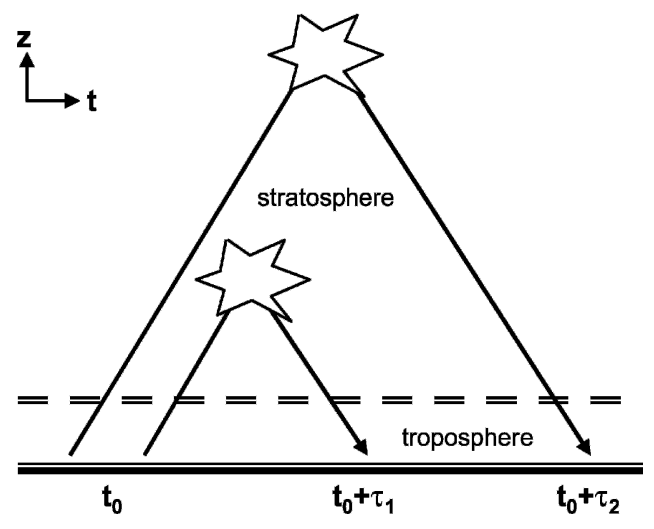

FIG. 12. Schematic illustration of how the timing of the tropospheric response is determined by the level of maximum eddy driving in the stratosphere.

tions, so that the signal arrives later in the troposphere if it is initially stimulated at higher levels in the stratosphere.

Just what controls the rate of downward descent of TST signals in this model and in the observations remains an open question. However, for realistic values of stratospheric thermal damping, we know that the rate of descent of TST signals should increase as the damping rate is increased. Two effects might be at work here, first, the fact that the phase speed of the linear adjustment of the zonal-mean circulation to stratospheric perturbations is proportional to the damping rate (Dickinson 1968; Haynes et al. 1991; KP), and second that the eddy driving of the mean flow is stronger if the damping rate is stronger (as in the weakly nonlinear theory of the quasibiennial oscillation). Sorting out these issues is beyond the scope of this study, but to support the idea that the rate of downward descent is an increasing function of the damping rate, we show in Fig. 13 the results of additional perturbation-ensemble realizations in which the stratospheric damping rates are doubled from $1 /(20$ day) to $1 /(10$ day $)$, and halved from $1 /(20$ day $)$ to $10 /(40$ day $)$. The figure shows the frequency distribution of $\mathrm{E}, \mathrm{I}$, and $\mathrm{L}$ cases, and indeed supports the idea that stronger damping rates favor earlier tropospheric return signals, and weak damping rates favor later tropospheric return signals.

We also note interesting similarities between this work and a recent observational study of stratospheric sudden warmings (SSWs) by Limpasuvan et al. (2004). As in the observations, we find that the downward propagation in the model is linked to EP-flux anomalies in higher wavenumbers as the signal reaches the troposphere (not shown). The signature of the preconditioned upper stratosphere with a dipole pattern in the 




FIG. 13. The frequency distribution of E, I, and L cases for different values of the stratospheric damping rate $k_{s}$. The distributions for $k_{s}=1 /(10$ day $)$ and 1/(40 day) are derived from additional perturbation experiments with ensemble sizes of 101, and the distribution for $k_{s}=1 /(20$ day $)$ comes from the standard perturbation experiment of this study (Fig. 6). As in Fig. 6, realizations with a large initial AM are excluded for the calculation of the corresponding distribution.

zonal wind and a positive temperature anomaly over the Pole (Fig. 8, right panels) is also similar to the observations. Lastly, we find that cold anomalies develop in the upper stratosphere after the initial forcing period (e.g., Fig. 4d), a feature that can also be seen from observations (Limpasuvan et al. 2004, their Fig. 4).

Finally, we note that one of the most surprising findings of the present study is the large variability of the response to surface perturbations, as exemplified by Fig. 4, especially in light of our previous experience with generating upward propagating waves by perturbing the lower stratosphere (Polvani and Saravanan 2000; Scott et al. 2004). This variability is caused in the present study by the way the pulse interacts with the tropospheric circulation on the way to the stratosphere, and by the sensitivity of the stratospheric breaking to details of the polar-vortex structure. Given this uncertainty in the atmospheric response at early times (days $0-20$ ), it is perhaps not surprising that the response at later times was found to be so highly variable.

Acknowledgments. The authors thank N. Harnik, J. Perlwitz, W. Robinson, and T. Shepherd for useful discussions. We also appreciate the comments of three anonymous reviewers, which helped to improve the final draft of this paper. PJK acknowledges the support of the Natural Sciences and Research Council of Canada. LMP is supported, in part, by a grant from the U.S. National Science Foundation. TJR would like to thank GFDL and the Atmospheric and Oceanic Sciences Program at Princeton University for their generous support.

\section{APPENDIX}

\section{Forcing of the Model}

We apply linear Rayleigh damping to the momentum equations using a damping coefficient $k_{v}$ of

$$
k_{v}(\sigma)= \begin{cases}k_{f} \frac{\sigma-\sigma_{b}}{1-\sigma_{b}}, & \sigma>\sigma_{b} \\ k_{\mathrm{sp}}\left(\frac{\sigma_{\mathrm{sp}}-\sigma}{\sigma_{\mathrm{sp}}}\right)^{2}, & \sigma<\sigma_{\mathrm{sp}} \\ 0, & \text { else. }\end{cases}
$$

Following HS, we chose $\sigma_{b}=0.7(\sim 700 \mathrm{hPa})$ and $k_{f}=$ 1 day $^{-1}$ to mimic frictional effects in the planetary boundary layer. A sponge layer with $\sigma_{\mathrm{sp}}=5 \times 10^{-4}$ $(\sim 0.5 \mathrm{hPa})$ and $k_{\mathrm{sp}}=0.5$ day $^{-1}$ is added at the model top to simulate gravity wave drag in the mesosphere and to prevent spurious wave reflection from the upper boundary.

We use Newtonian cooling to relax the temperatures $T$ toward a prescribed equilibrium temperature profile $T_{\text {eq }}$ :

$$
\frac{\partial T}{\partial t}=\ldots-k_{T}(\phi, \sigma)\left[T-T_{\mathrm{eq}}(\phi, p)\right]
$$

with a thermal relaxation coefficient $k_{T}$ of

$k_{T}(\phi, \sigma)= \begin{cases}k_{a}+\left(k_{s}-k_{a}\right) \frac{\sigma-\sigma_{b}}{1-\sigma_{b}} \cos ^{4} \phi, & \sigma \geq \sigma_{b} \\ k_{a}, & \sigma_{b}>\sigma \geq \sigma_{s 1} \\ k_{\mathrm{st}}+\left(k_{a}-k_{\mathrm{st}}\right) \frac{\sigma-\sigma_{s 2}}{\sigma_{s 1}-\sigma_{s 2}}, & \sigma_{s 1}>\sigma \geq \sigma_{s 2} \\ k_{\mathrm{st}}, & \sigma_{s 2}>\sigma .\end{cases}$

In the troposphere $\left(\sigma \geq \sigma_{s 1}\right)$ we use $k_{a}=1 /(40$ day $)$ and $k_{s}=1 /(4$ day) as in the HS prescription. To match more closely observed stratospheric values (Dickinson 1968), we modify this prescription in the stratosphere so that $k_{T}$ transitions as a linear function of sigma to a value of $k_{\mathrm{st}}=1 /(20$ day $)$ between $\sigma_{s 1}=0.15(\sim 150 \mathrm{hPa})$ and $\sigma_{s 2}=0.095(\sim 95 \mathrm{hPa})$, and $k_{T}=k_{\mathrm{st}}=1 /(20$ day $)$ above.

Following PK and KP, the equilibrium temperature profile $T_{\text {eq }}$ is defined by

$$
T_{\mathrm{eq}}(\phi, p)= \begin{cases}T_{\mathrm{eq}}^{\mathrm{strat}}(\phi, p), & p<p_{T} \\ T_{\mathrm{eq}}^{\mathrm{trop}}(\phi, p), & p \geq p_{T}\end{cases}
$$


with latitude $\varphi$, pressure $p$, and a tropopause at $p_{T}=$ $100 \mathrm{hPa}$. The stratospheric relaxation temperature is given by

$$
T_{\mathrm{eq}}^{\mathrm{strat}}(\phi, p)=[1-W(\phi)] T_{\mathrm{US}}(p)+W(\phi) T_{\mathrm{PV}}(p ; \gamma),
$$

where $T_{\mathrm{US}}(p)$ is taken from the temperature profile of the U.S. Standard Atmosphere 1976 (COESA 1976), and

$$
T_{\mathrm{PV}}(p ; \gamma)=T_{\mathrm{US}}\left(p_{T}\right)\left(p / p_{T}\right)^{(R \gamma / g)}
$$

is the temperature of an atmosphere with constant lapse rate $\gamma$, and standard notation otherwise. The parameter $\gamma$, which controls the strength of the stratospheric temperature gradient over the winter pole (PK), is set to $1 \mathrm{~K} \mathrm{~km}^{-1}$. The weight function

$$
W(\phi)=\frac{1}{2}\left[1+\tanh \left(\frac{\phi-\phi_{0}}{\Delta \phi}\right)\right],
$$

is used to confine the cooling over the winter pole. With $\varphi_{0}=50^{\circ} \mathrm{N}$ and $\Delta \varphi=10^{\circ} \mathrm{N}$ latitude, $T_{\mathrm{eq}}^{\text {strat }}$ varies smoothly from a cold stratosphere over the high latitudes of the winter (northern) hemisphere to the U.S. Standard Atmosphere 1976 over all other latitudes. The tropospheric relaxation temperature $T_{\mathrm{eq}}^{\mathrm{trop}}$ is defined by

$$
T_{\mathrm{eq}}^{\mathrm{trop}}(\phi, p)=\max \left[T_{\mathrm{US}}\left(p_{T}\right),\left(T_{0}-\delta T\right)\left(p / p_{0}\right)^{\kappa}\right],
$$

where $T_{0}=315 \mathrm{~K}, p_{0}=1000 \mathrm{hPa}$, and $\kappa=2 / 7$, with

$$
\delta T=\delta_{y} \sin ^{2} \phi+\varepsilon \sin \phi+\delta_{z} \cos ^{2} \phi \log \left(p / p_{0}\right),
$$

where $\delta_{y}=60 \mathrm{~K}, \delta_{z}=10 \mathrm{~K}$, and $\varepsilon=0$. With this choice of parameters the tropospheric equilibrium temperature profile is identical to the HS benchmark.

\section{REFERENCES}

Ambaum, M. H. P., and B. J. Hoskins, 2002: The NAO troposphere-stratosphere connection. J. Climate, 15, 1969-1978.

Andrews, D. G., J. R. Holton, and C. B. Leovy, 1987: Middle Atmosphere Dynamics. Academic Press, 489 pp.

Baldwin, M. P., and T. J. Dunkerton, 1989: The stratospheric major warming of early December 1987. J. Atmos. Sci., 46, 28632884.

- and — 1999: Propagation of the Arctic Oscillation from the stratosphere to the troposphere. J. Geophys. Res., 104, 30 937-30 946.

—, and —, 2001: Stratospheric harbingers of anomalous weather regimes. Science, 294, 581-584.

—, D. B. Stephenson, D. W. J. Thompson, T. J. Dunkerton, A. J. Charlton, and A. O'Neill, 2003a: Stratospheric memory and extended-range weather forecasts. Science, 301, 636-640.

—, D. W. J. Thompson, E. F. Shuckburgh, W. A. Norton, and
N. P. Gillett, 2003b: Weather from the stratosphere? Science, 301, 317-319.

Black, R. X., 2002: Stratospheric forcing of surface climate in the Arctic Oscillation. J. Climate, 15, 268-277.

Charlton, A. J., A. O. O'Neill, W. A. Lahoz, and A. C. Massacand, 2004: Sensitivity of tropospheric forecasts to stratospheric initial conditions. Quart. J. Roy. Meteor. Soc., 130, 1771-1792.

Charney, J. G., and P. G. Drazin, 1961: Propagation of planetaryscale disturbances from the lower into the upper atmosphere. J. Geophys. Res., 66, 83-109.

Christiansen, B., 2003: Temporal growth and vertical propagation of perturbations in the winter atmosphere. Quart. J. Roy. Meteor. Soc., 129, 1589-1606.

COESA, 1976: U.S. Standard Atmosphere. U.S. Government Printing Office, $227 \mathrm{pp}$.

Cohen, J., D. Salstein, and K. Saito, 2002: A dynamical framework to understand and predict the major Northern Hemisphere mode. Geophys. Res. Lett., 29, 1412, doi:10.1029/ $2001 \mathrm{GL} 014117$.

Dickinson, R. E., 1968: On the excitation and propagation of zonal winds in an atmosphere with Newtonian cooling. $J$. Atmos. Sci., 25, 269-279.

Garric, G., S. A. Venegas, C. E. Tansley, and I. N. James, 2003: Atmosphere-sea-ice low-frequency variability with a simple model of the Southern Hemisphere. Quart. J. Roy. Meteor. Soc., 129, 2347-2366.

Gillett, N. P., and D. W. J. Thompson, 2003: Simulation of recent Southern Hemisphere climate change. Science, 302, 273-275.

Haynes, P. H., M. E. McIntyre, T. G. Shepherd, C. J. Marks, and K. P. Shine, 1991: On the "downward control" of extratropical diabatic circulations by eddy-induced mean zonal forces. J. Atmos. Sci., 48, 651-680.

Held, I. M., and M. J. Suarez, 1994: A proposal for the intercomparison of the dynamical cores of atmospheric general circulation models. Bull. Amer. Meteor. Soc., 75, 1825-1830.

Holton, J. R., and C. Mass, 1976: Stratospheric vacillation cycles. J. Atmos. Sci., 33, 2218-2225.

James, I. N., and P. M. James, 1989: Ultra-low frequency variability in a simple atmospheric circulation model. Nature, 342, 53-55.

Kushner, P. J., and L. M. Polvani, 2004: Stratosphere-troposphere coupling in a relatively simple AGCM: The role of eddies. $J$. Climate, 17, 629-639.

Limpasuvan, V., D. Thompson, and D. L. Hartmann, 2004: The life cycle of the Northern Hemisphere sudden stratospheric warmings. J. Climate, 17, 2584-2596.

Matsuno, T., 1971: A dynamical model of the stratospheric sudden warming. J. Atmos. Sci., 28, 1479-1494.

McIntyre, M. E., and T. N. Palmer, 1984: The "surf zone" in the stratosphere. J. Atmos. Terr. Phys., 46, 825-849.

Perlwitz, J., and N. Harnik, 2003: Observational evidence of a stratospheric influence on the troposphere by planetary wave reflection. J. Climate, 16, 3011-3026.

Plumb, R. A., and K. Semeniuk, 2003: Downward migration of extratropical zonal wind anomalies. J. Geophys. Res., 108, 4223, doi:10.1029/2002JD002773.

Polvani, L. M., and R. Saravanan, 2000: The three-dimensional structure of breaking Rossby waves in the polar wintertime stratosphere. J. Atmos. Sci., 57, 3663-3685.

— and P. J. Kushner, 2002: Tropospheric response to strato- 
spheric perturbations in a relatively simple general circulation model. Geophys. Res. Lett., 29, 1114, doi:10.1029/ 2001 GL014284.

— , and D. W. Waugh, 2004: Upward wave activity flux as precursor to extreme stratospheric events and subsequent anomalous surface weather regimes. J. Climate, 17, 35483554.

Scinocca, J. F., and P. H. Haynes, 1998: Dynamical forcing of stratospheric planetary waves by tropospheric baroclinic eddies. J. Atmos. Sci., 55, 2361-2392.
Scott, R. K., D. G. Dritschel, L. M. Polvani, and D. W. Waugh, 2004: Enhancement of Rossby wave breaking by steep potential vorticity gradients in the winter stratosphere. J. Atmos. Sci., 61, 904-918.

Song, Y., and W. A. Robinson, 2004: Dynamical mechanism for stratospheric influences on the troposphere. J. Atmos. Sci., 61, 1711-1725.

Thompson, D. W. J., and S. Solomon, 2002: Interpretation of recent Southern Hemisphere climate change. Science, 296, 895-899. 